\title{
Entrapment of Permanent Pacemaker Lead as the Cause of Tricuspid Regurgitation Role of 3-Dimensional Echocardiography
}

\author{
Tien-En Chen, MD; Chun-Chieh Wang, MD; \\ Ming-Shyan Chern, MD; Jaw-Ji Chu, MD*
}

\begin{abstract}
Transthoracic 2-dimensional (D) echocardiography (echo) is often used to assess tricuspid regurgitation (TR) after implantation of permanent pacemakers. However, its ability to define the precise anatomical relationship between the tricuspid valve and the pacemaker lead is quite limited. This report presents a 58-year-old male with aggravation of TR after pacemaker implantation for heart block. Three-D echo precisely depicted the entrapment of the lead shaft in the fused and fibrotic septal and posterior tricuspid leaflets. The patient underwent tricuspid valve annuloplasty and the symptoms of right heart failure improved soon after the operation. (Circ J 2007; 71: 1169-1171)
\end{abstract}

Key Words: Entrapment; Pacemaker lead; Three-dimensional echocardiography; Tricuspid regurgitation

$\mathbf{R}$ ight heart failure because of tricuspid regurgitation (TR) after permanent pacemaker (PPM) has been reported!-6 Conventional 2-dimensional (D) echocardiography (echo), though very important for detecting TR, is quite limited in depicting the precise anatomical relationship between the tricuspid leaflets and PPM lead shaft? This relationship may determine the pathogenesis of TR and realization of it may help determine the policy of management. In this report, with the assistance of 3D echo, the structure of the tricuspid valve, its incompetence and the entrapment of the PPM lead shaft in the valve were precisely delineated.

\section{Case Report}

A 58-year-old man underwent implantation of a transvenous VVIR PPM for atrial fibrillation and complete heart block 9 years ago. Prior to PPM, echocardiography showed moderate TR, with a TR area of $16.6 \mathrm{~cm}^{2}$ and a right atrial (RA) area of $28.8 \mathrm{~cm}^{2}$, and normal systolic function of both ventricles. Three years after PPM implantation, 2D echo showed aggravation of TR with a TR area of $19.4 \mathrm{~cm}^{2}$ and a RA area of $37.4 \mathrm{~cm}^{2}$. Since then, he had abdominal fullness and edema of both legs. These symptoms gradually became aggravated. Follow-up transthoracic echocardiography was performed by using a Philips SONOS 7500 (Philips Medical Systems, Eindhoven, Netherlands) and pyramid-shaped full-volume $3 \mathrm{D}$ images of the right ventricle $(\mathrm{RV})$ were acquired from between the left parasternal border and the apical window with a $4 \mathrm{MHz}$ and $4 \times$ matrix transducer under ECG gating while the patient gently held his breath. The 2D echo revealed slightly impaired systolic function of the left ventricle with abnormal septal wall motion and mild

(Received January 18, 2007; revised manuscript received April 5, 2007; accepted April 11, 2007)

Second Section of Cardiology, Department of Internal Medicine, *Division of Cardiovascular Surgery, Chang Gung Memorial Hospital, Linkou Medical Center, Taoyuan, Taiwan

Mailing address: Ming-Shyan Chern, MD, Chang Gung Memorial Hospital, No 5, Fushing Street, Gueshian, Taoyuan, Taiwan. E-mail: mschern@adm.cgmh.org.tw mitral regurgitation. The RV, RA and inferior vena cava were dilated, and RV function was normal (Fig 1A). Tethering of the septal leaflet of tricuspid valve was observed, together with no coaptation of thes leaflets, thus causing severe TR (Fig 1B). The maximal area of TR was $42.1 \mathrm{~cm}^{2}$ and that of RA $64.6 \mathrm{~cm}^{2}$. The transtricuspid systolic pressure gradient was $12 \mathrm{mmHg}$. However, the relationship between the tricuspid valve and the PPM lead could not be observed on the multiple views of 2D echo (Figs 1C,D).

After being cropped in from the apex toward the base, the full-volume 3D echo showed that the shaft of the PPM lead was entrapped in the thickened, fibrotic and fused posterior and septal leaflets (Fig 2A), which could not coapt during systole, and the vena contracta was well seen (Fig 2A, arrowhead). During diastole, the anterior tricuspid leaflet opened freely. The anterior two-thirds of the septal leaflet was tethered, but not fixed, and could be pushed open slightly. The remaining one-third of the septal leaflet and the entire posterior leaflet was thickened, tethered and frozen (Fig 2B). When the full-volume 3D pyramid was cropped in from the RV free wall, the entrapment of the PPM shaft in the fused septal and posterior leaflets and the free movements of anterior tricuspid leaflet were noted clearly during the cardiac cycle (Figs 2C,D).

During operation, the PPM lead was found to be encapsulated in fibrotic tissue and severely adherent to the fused commissural areas of the septal and posterior leaflets (Fig 3A). Additionally, there was a very small atrialized area of the basal septal part of the RV, indicative of a mild form of Ebstein anomaly (Fig 3A: asterisk). Tricuspid valve repair was performed with partial plication of the atrialized $\mathrm{RV}$ in the septal region, followed by annuloplasty (Fig 3B). The repeated 2D echo showed good coaptation of tricuspid leaflets with mild TR at 1 week after operation. Symptoms of right-heart failure improved significantly after operation and the patient was discharged 2 weeks later.

\section{Discussion}

Significant TR because of tricuspid malfunction caused 

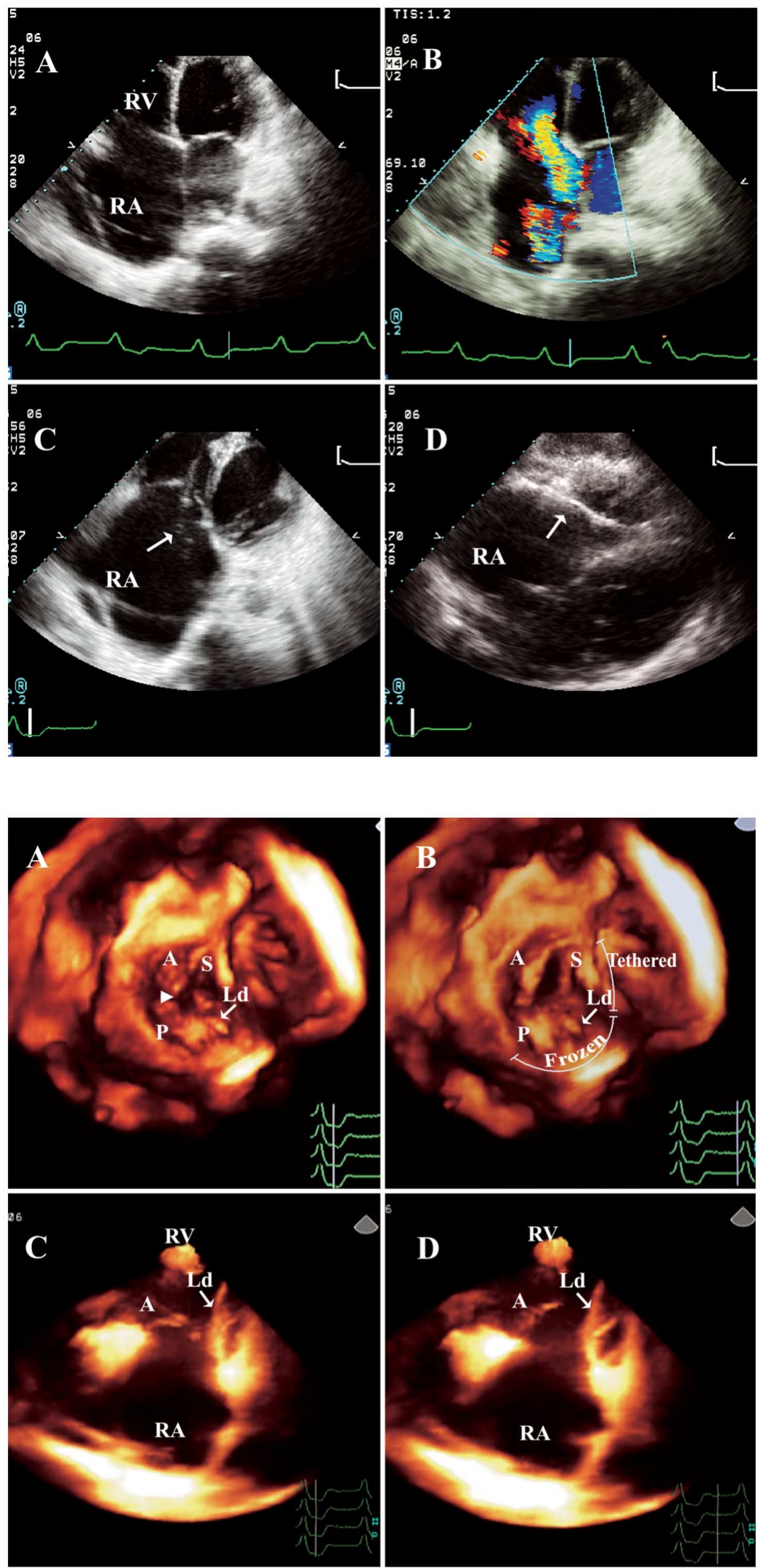

Fig 1. (A) Apical 4-chamber view from 2-dimensional echocardiography shows a dilated right atrium (RA) and right ventricle (RV). (B) Color flow Doppler showing severe tricuspid regurgitation caused by tethering of the septal leaflet and no coaptation of the tricuspid leaflets. (C) Modified 4-chamber view and (D) subcostal view are unable to show the structure of the whole tricuspid valve and its relationship with the pacemaker lead (white arrow).

Fig 2. Three-dimensional (3D) echocardiography cropped and viewed from the right ventricular apex and right ventricular free wall. (A) Stop-frame at mid-systole of 3D image cropped in from right ventricle (RV) apex: showing that the pacemaker lead is encapsulated by thickened, fibrotic and fused septal and posterior leaflets of the tricuspid valve. Arrowhead points to the vena contracta of the tricuspid regurgitation. (B) Stop-frame at late-diastole of 3D image cropped in from RV apex: showing that the anterior leaflet is moving freely while the anterior two-thirds of the septal leaflet is tethered; the remaining septal leaflet and the posterior leaflet are fused and frozen. (C, D) Stop-frames at mid-systole and mid-diastole of 3D images cropped in from the RV free wall showing free movement of the anterior leaflet and entrapment of the pacemaker lead in the septal and posterior leaflets. A, anterior leaflet; $\mathrm{P}$, posterior leaflet; S, septal leaflet; Ld, lead; $\mathrm{RA}$, right atrium. 

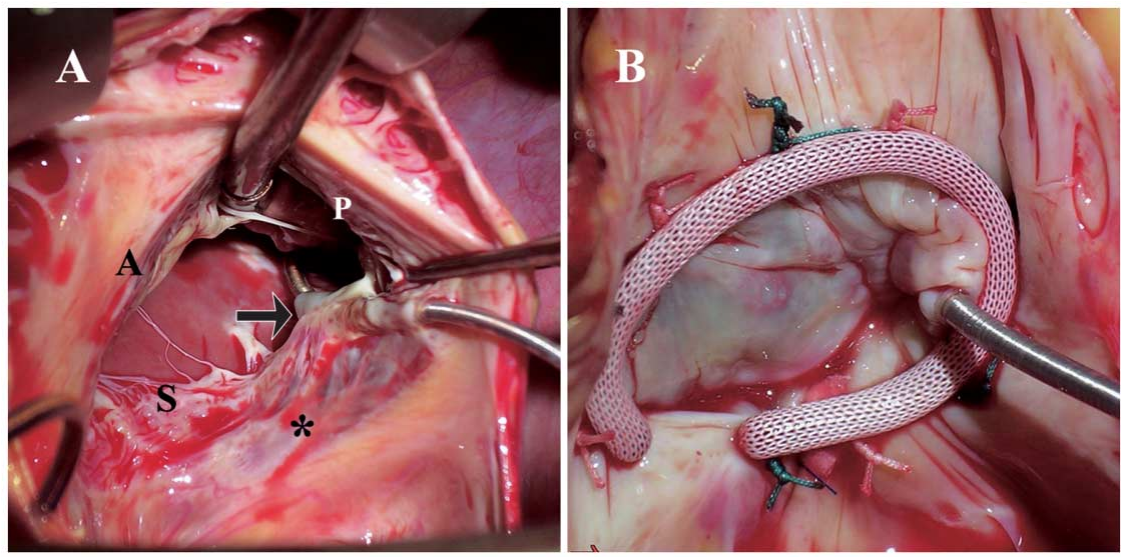

Fig 3. (A) Operative finding showing the pacemaker lead (black arrow) encapsulated by the fibrotic and fused posterior and septal leaflets. *Small flattened area of atrialized right ventricle. (B) Partial plication of septal leaflet and annuloplasy ensure good coaptation of the 3 leaflets. A, anterior leaflet; P, posterior leaflet; $\mathrm{S}$, septal leaflet.

by PPM leads is occasionally observed, ${ }^{1-5}$ although its true incidence is unknown. The mechanisms of PPM-related TR include leaflet perforation or laceration, interference of coaptation by the lead, asynchronous RV activation from apex to base, and entrapment and encapsulation of the PPM lead by scar tissue ${ }^{-6}$

Panigua et $\mathrm{al}^{5}$ have reported an increased incidence of moderate-to-severe TR in patients with transvenous PPM compared with age- and sex-matched controls, and they suggested that PPM lead may aggravate TR. This phenomenon was noted in the present patient, who had moderate TR before placement of the PPM and in whom the symptoms of right-heart failure appeared gradually 6 years later.

The management of this tricuspid malfunction includes removal of the PPM leads by extraction 7,8 and surgery? McCarthy et al have reported a 30-day operative mortality of $6 \%$ for tricuspid valve replacement? Therefore, surgery for TR caused by a PPM lead remains controversial and depends on the clinical situation. However, the nonsurgical removal of a chronic PPM lead is risky and sometimes fatal. Nonfatal complications, such as ventricular arrhythmia, lead fragmentation, myocardial avulsion, hemopericardium and pulmonary embolism can occur? Most of the complications are associated with the difficulty of emancipating the leads from adhesion or scar tissue? Therefore, it would be enormously helpful for the therapeutic decisionmaking process to delineate the cardiac structure as well as the PPM lead, particularly around the tricuspid leaflets.

Using conventional transthoracic 2D echo, Lin et al found that the PPM-caused tricuspid malfunction could only be diagnosed preoperatively in 5 of 41 patients (12\%) who underwent operation for PPM-related TR? That means that conventional transthoracic $2 \mathrm{D}$ echo is quite limited in its ability to assess tricuspid structure and its relationship to the PPM leads. The visualization of the tricuspid valve on a 2D echo can be difficult because only 2 leaflets can be shown simultaneously in the atypical parasternal view and the posterior leaflet could only be seen on the RV 2-chambered view. Schnabel et al have shown that transthoracic real-time 3D echo enabled better assessment of the whole tricuspid valve, with a satisfactory inter-rater agreement ${ }^{10}$

In the present study, the morphology of the whole tricuspid valve could not be depicted well on $2 \mathrm{D}$ echo. Although we attempted to trace the entire path of the PPM lead from various views, the relationship between the lead and the tricuspid apparatus remained unidentifiable. Fortunately, real-time 3D echo provided excellent visualization of the tricuspid leaflets and the lead. By cropping the full-volume imaging pyramid in from the RV apex toward the base and RV free wall, the following were clearly observed: (1) the encapsulation and entrapment of the PPM lead by thick and fibrotic septal and posterior tricuspid leaflets, (2) the structure of the whole tricuspid valve and the movement of each leaflet and (3) the incompetent area of the tricuspid valve. The patient was sent for surgery based on these findings, which were all confirmed at operation.

\section{Conclusion}

In addition to the anatomical and hemodynamic information obtained from conventional 2D echo, this case report has shown that real-time $3 \mathrm{D}$ echo can be very valuable in cases of PPM-related TR for depicting both the anatomical structure of the tricuspid valve and its relationship with the PPM lead. Such an assessment also contributes significantly to decision-making for further management.

\section{References}

1. Iskandar SB, Ann Jackson S, Fahrig S, Mechleb BK, Garcia ID. Tricuspid valve malfunction and ventricular pacemaker lead: Case report and review of the literature. Echocardiography 2006; 23: 692-697.

2. Lin G, Nishimura RA, Connolly HM, Dearani JA, Sundt TM 3rd, Hayes DL. Severe symptomatic tricuspid valve regurgitation due to permanent pacemaker or implantable cardioverter-defibrillator leads. J Am Coll Cardiol 2005; 45: 1672-1675.

3. Gould L, Reddy CV, Yacob U, Teich M, DeMartino A, DePalma D, et al. Perforation of the tricuspid valve by a transvenous pacemaker. JAMA 1974; 230: 86-87.

4. Fishenfeld J, Lamy Y. Laceration of the tricuspid valve by a pacemaker wire. Chest 1972; 61: 697-698.

5. Paniagua D, Aldrich HR, Lieberman EH, Lamas GA, Agatston AS. Increased prevalence of significant tricuspid regurgitation in patients with transvenous pacemakers leads. Am J Cardiol 1998; 82: 11301132 .

6. Huang TY, Baba N. Cardiac pathology of transvenous pacemakers. Am Heart J 1972; 83: 469-474.

7. Smith HJ, Fearnot NE, Byrd CL, Wilkoff BL, Love CJ, Sellers TD. Five-years experience with intravascular lead extraction: US Lead Extraction Database. Pacing Clin Electrophysiol 1994; 17: 2016-2020.

8. Kozlowski D, Dubaniewicz A, Kozluk E, Grzybiak M, Krupa W, Kolodziej P, et al. The morphological conditions of the permanent pacemaker lead extraction. Folia Morphol (Warsz) 2000; 59: 25-29.

9. McCarthy PM, Bhudia SK, Rajeswaran J, Hoercher KJ, Lytle BW, Cosgrove DM, et al. Tricuspid valve repair: Durability and risk factors for failure. J Thorac Cardiovasc Surg 2004; 127: 674-685.

10. Schnabel R, Khaw AV, von Bardeleben RS, Strasser C, Kramm T, Meyer J, et al. Assessment of the tricuspid valve morphology by transthoracic real-time-3D-echocardiography. Echocardiography 2005; 22: $15-23$. 